\title{
Radiotherapy and temozolomide for newly diagnosed glioblastoma and anaplastic astrocytoma: validation of Radiation Therapy Oncology Group-Recursive Partitioning Analysis in the IMRT and temozolomide era
}

\author{
Anthony J. Paravati · Dwight E. Heron · \\ Douglas Landsittel • John C. Flickinger • \\ Arlan Mintz • Yi-Fan Chen • M. Saiful Huq \\ Received: 2 September 2010/Accepted: 3 December 2010/Published online: 22 December 2010 \\ (C) The Author(s) 2010. This article is published with open access at Springerlink.com
}

\begin{abstract}
Since the development of the Radiation Therapy Oncology Group-Recursive Partitioning Analysis (RTOGRPA) risk classes for high-grade glioma, radiation therapy in combination with temozolomide (TMZ) has become standard care. While this combination has improved survival, the prognosis remains poor in the majority of patients. Therefore, strong interest in high-grade gliomas from basic research to clinical trials persists. We sought to evaluate whether the current RTOG-RPA retains prognostic
\end{abstract}

A. J. Paravati · D. E. Heron ( () J. C. Flickinger · A. Mintz ·

M. S. Huq

Department of Radiation Oncology, University of Pittsburgh Cancer Institute, 5230 Centre Ave, Pittsburgh, PA 15232, USA e-mail: herond2@upmc.edu

D. Landsittel

Department of Internal Medicine, University of Pittsburgh

Medical Center, Pittsburgh, PA, USA

J. C. Flickinger · A. Mintz

Department of Neurological Surgery, University of Pittsburgh

Cancer Institute, Pittsburgh, PA, USA

Y.-F. Chen

Department of Biostatistics, University of Pittsburgh Cancer Institute, Pittsburgh, PA, USA

D. E. Heron

Department of Otolaryngology, University of Pittsburgh Cancer Institute, Pittsburgh, PA, USA

A. J. Paravati - D. E. Heron - D. Landsittel ·

J. C. Flickinger - A. Mintz - Y.-F. Chen - M. S. Huq

University of Pittsburgh School of Medicine, Pittsburgh, PA, USA

D. E. Heron

Department of Radiation Oncology, University of Pittsburgh Medical Centre, 5230 Centre Ave, Pittsburgh, PA 15232, USA significance in the TMZ era or alternatively, if modifications better prognosticate the optimal selection of patients with similar baseline prognosis for future clinical protocols. The records of 159 patients with newly-diagnosed glioblastoma (GBM, WHO grade IV) or anaplastic astrocytoma (AA, WHO grade III) were reviewed. Patients were treated with intensity-modulated radiation therapy (IMRT) and concurrent followed by adjuvant TMZ $(n=154)$ or adjuvant TMZ only $(n=5)$. The primary endpoint was overall survival. Three separate analyses were performed: (1) application of RTOG-RPA to the study cohort and calculation of subsequent survival curves, (2) fit a new tree model with the same predictors in RTOG-RPA, and (3) fit a new tree model with an expanded predictor set. All analyses used a regression tree analysis with a survival outcome fit to formulate new risk classes. Overall median survival was 14.9 months. Using the RTOG-RPA, the six classes retained their relative prognostic significance and overall ordering, with the corresponding survival distributions significantly different from each other $\left(P<0.01, \chi^{2}\right.$ statistic $\left.=70\right)$. New recursive partitioning limited to the predictors in RTOG-RPA defined four risk groups based on Karnofsky Performance Status (KPS), histology, age, length of neurologic symptoms, and mental status. Analysis across the expanded predictors defined six risk classes, including the same five variables plus tumor location, tobacco use, and hospitalization during radiation therapy. Patients with excellent functional status, AA, and frontal lobe tumors had the best prognosis. For patients with newly-diagnosed high-grade gliomas, RTOGRPA classes retained prognostic significance in patients treated with TMZ and IMRT. In contrast to RTOG-RPA, in our modified RPA model, KPS rather than age represented the initial split. New recursive partitioning identified potential modifications to RTOG-RPA that should be further explored with a larger data set. 
Keywords Glioblastoma - Anaplastic Astrocytoma . RTOG-RPA · Validation · Temozolomide · IMRT

\section{Introduction}

High-grade gliomas, glioblastoma (GBM) and anaplastic astrocytoma (AA), are the most common and most malignant brain tumors in adults. Prior to the development of temozolomide (TMZ), the survival of patients with highgrade gliomas had not improved significantly. Extent of surgical resection and adjuvant radiation have been shown in several clinical trials from the 1970s to the 1990s to be the best predictors of outcomes, leading to wide recognition of these modalities as standard therapy [1]. However, trials of adjuvant chemotherapy in the pre-temozolomide era were equivocal, including a meta-analysis of 12 randomized clinical trials that showed only a small survival benefit [2]. Over the past decade, several trials of novel biologic or other therapeutic interventions, excluding those of TMZ, have shown little impact on survival compared to the pretreatment and treatment-related factors described in the recursive partitioning analysis by Curran et al. [3, 4]. The division of high-grade glioma patients into prognostic groups formed from recursive partitioning across these pretreatment and treatment-related prognostic variables has proven valuable in myriad clinical trials [4-14].

For patients treated with radiation therapy only, the Radiation Therapy Oncology Group-Recursive Partitioning Analysis (RTOG-RPA) was developed to obtain groups of patients with similar survival times [3]. Because the survival of high-grade glioma patients remains relatively poor, there has been a sustained interest in developing phase II protocols. RTOG-RPA has been used extensively in the design and interpretation of phase II protocols and for refining stratification in phase III trials [5, 6, 8-17].

The principle advantage of using a recursive partitioning model to analyze survival is that tree models yield a more clinically interpretable result, and interactions and nonlinearity are implicitly fit in the model. While the proportional hazards model provides relative risks based on patient characteristics, it does not translate into defined survival-based risk classes amendable to evaluating the efficacy of a therapy.

Prior to the TMZ era, RTOG-RPA was validated in two published series [4, 7]. However in recent years, TMZ was repeatedly shown to unequivocally improve overall survival of patients when given concurrently with radiation therapy and as adjuvant [16-18]. The acceptance of TMZ as standard treatment for high-grade glioma patients was a significant change in therapy following the development of the RTOG-RPA in 1993. In this context, we have conducted a validation study of RTOG-RPA to determine if it retains prognostic value in high-grade glioma patients treated with TMZ in addition to intensity-modulated radiation therapy (IMRT) in patients with GBM and AA. We also report modifications to the existing RTOG-RPA that increased its prognostic value in our patients.

\section{Methods}

This study was approved by the University of Pittsburgh Cancer Institute's Institutional Review Board. One hundred seventy-six (176) patients with newly-diagnosed high-grade glioma who were treated with IMRT generated from Eclip$\mathrm{se}^{\mathrm{TM}}$ TPS (Varian Medical Systems, Palo Alto, CA) and TMZ between March 2004 and March 2009 were identified. In all cases, diagnosis was established by pathology review at the University of Pittsburgh Medical Centers. Patients with cerebellar $(n=6)$, brainstem $(n=5)$, pineal $(n=3)$ or optic tumors $(n=3)$ were excluded from further analysis. The remaining 159 patients were treated with IMRT and concurrent followed by adjuvant TMZ $(n=154)$ or adjuvant TMZ only $(n=5)$. All data included in this analysis were extracted from the paper and electronic medical records with blinding to patients' date of death and last follow-up.

Three separate analyses were conducted. The first, validation of RTOG-RPA, was completed by assigning RTOG-RPA risk class to each patient in our cohort (Fig. 1). We subsequently determined if the Kaplan-Meier survival curves of RTOG-RPA risk classes were significantly different from each other using the HarringtonFleming test in R statistical software [19].

The second analysis again used only the covariates in RTOG-RPA. The breakpoints for all covariates in this analysis were also the same as in RTOG-RPA. However, recursive partitioning was newly conducted to produce subgroups of patients whose survival was as homogenous as possible based on our current data.

For the third and final analysis, we fit a tree model to our cohort using an expanded set of predictors, including those in RTOG-RPA. The complete list of these predictors includes age, race, Karnofsky Performance Status (KPS), histology (GBM or AA), neurologic functional classification (class 1: able to work or class 2: able to be at home and classes 3: hospitalized), surgery type (biopsy or Gross total resection/subtotal resection), IMRT dose, hospital admission during IMRT, IMRT treatment breaks, tumor location, the presence or absence of coexisting medical conditions (diabetes mellitus, hypertension, cardiac disease), tobacco use, duration of TMZ treatment, duration of 13 neurologic signs and symptoms, and the presence or absence of each neurologic symptom. Those neurologic signs and symptoms were cerebral deficit, cranial nerve deficit, memory lag, personality change, seizure history, sensory deficit, motor 


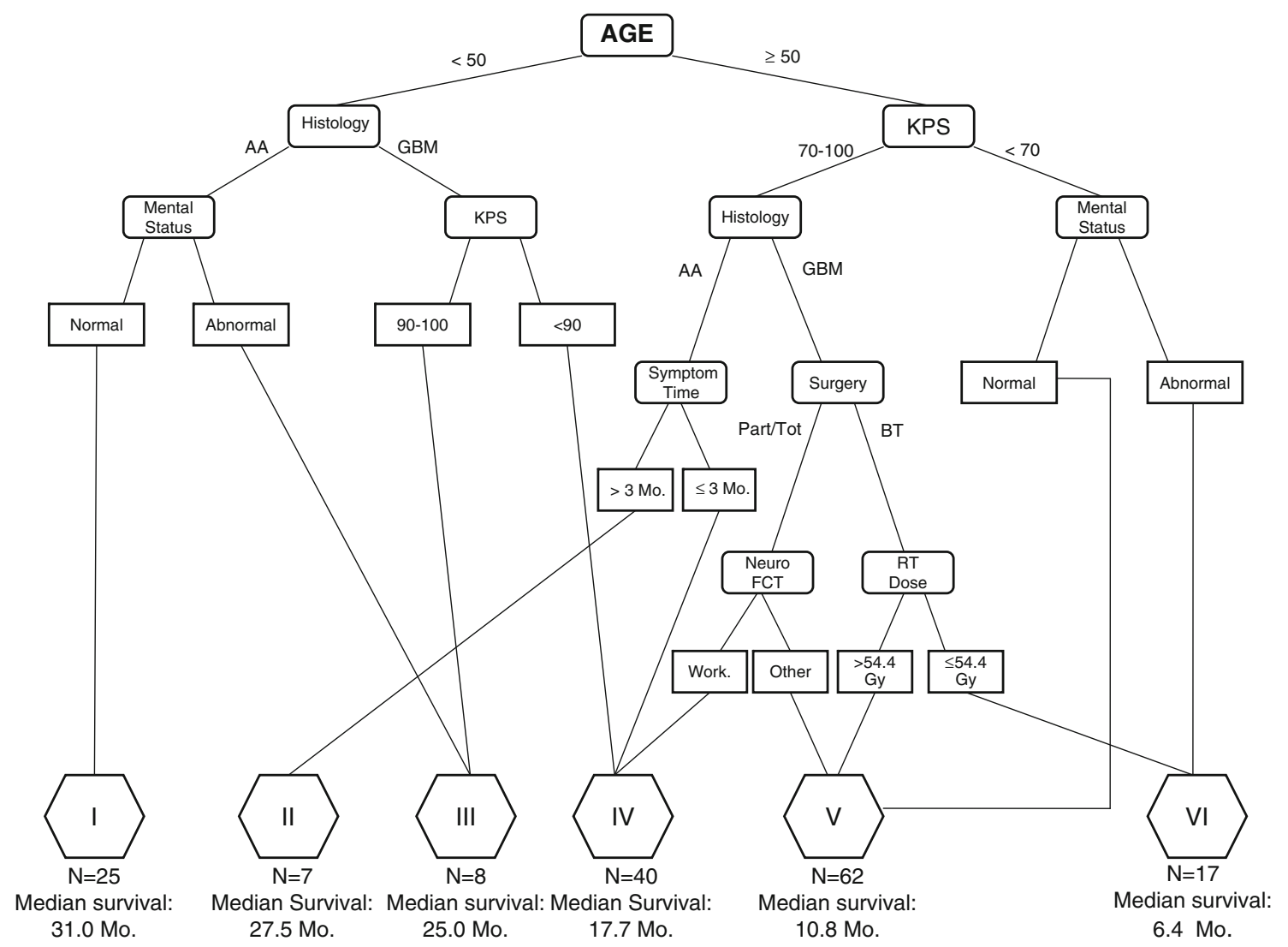

Fig. 1 Published RTOG-RPA displaying 12 terminal nodes combined into six significantly different survival classes $(P<0.01)$. Our survival results are displayed below the corresponding RTOG-RPA

deficit, papilledema, somnolence, speech impairment, headache, and mental status changes. Chemotherapy was not one of the treatment-related factors considered because all patients received TMZ.

The recursive partitioning model uses the rpart function in R statistical software [19] to identify the optimal split across all variables and across all possible cutoffs of each sorted variable. The optimality of a given split is judged using exponential scaling [20] to assess the log-likelihood defined by LeBlanc and Crowley [21]. Patients are then partitioned into the resulting subgroups, one with longer overall survival and one with shorter overall survival. This partitioning process is repeated until it produces terminal nodes that perfectly discriminate survival from death, or until the data cannot be partitioned further (i.e., $n<10$ within subgroup). It should be noted that the given method does not utilize significance tests or $P$-values, but rather represents a computational approach to estimate the final subsets which are as homogeneous as possible in terms of survival. Inclusion or exclusion of a given variable from the tree therefore does not represent the presence or absence of statistical significance. All statistical analyses were conducted with R statistical software. risk class. BT biopsy, Part/Tot subtotal resection/gross total resection, Neuro FCT neurological function

For terminal nodes resulting from recursive partitioning with similar survival distributions, we combined these nodes using the following algorithm: (1) order subgroups from shortest to longest median survival, (2) conduct the log-rank test between adjacent curves (i.e., with the most similar survival distributions), (3) combine nodes with nonsignificant results between them, unless they both originate from the same final split forming the given two terminal nodes, (4) reproduce the survival curves and related statistics (median survival, 2-year survival estimates) using the subsequently formed sub-groups. Overall survival outcomes in the combined recursively partitioned model were tested with the Harrington-Fleming test to determine if survival of the resulting risk classes were significantly different from each other.

\section{Results}

Patient and treatment demographics

Of the 176 patients treated with IMRT and TMZ between March 2004 and March 2009, 159 were evaluable for 
Table 1 Patient demographics and tumor characteristics

\begin{tabular}{|c|c|}
\hline Characteristic & Percent of assessable patients (no.) \\
\hline \multicolumn{2}{|l|}{ Gender } \\
\hline Men & $61(97)$ \\
\hline Women & $39(62)$ \\
\hline \multicolumn{2}{|l|}{ Age (years) } \\
\hline$<40$ & $19.4(31)$ \\
\hline $40-49$ & $15.7(25)$ \\
\hline $50-59$ & $30.2(48)$ \\
\hline$\geq 60$ & $34.6(55)$ \\
\hline \multicolumn{2}{|l|}{ Race } \\
\hline White & $94.3(150)$ \\
\hline Other & $5.7(9)$ \\
\hline \multicolumn{2}{|c|}{ Symptom duration (months) } \\
\hline$<2$ & $52.2(83)$ \\
\hline $2-4$ & $22.6(36)$ \\
\hline$\geq 4$ & $25.2(40)$ \\
\hline \multicolumn{2}{|c|}{ Neurological class } \\
\hline 1 Work & $28.3(45)$ \\
\hline 2 Home & $66.7(106)$ \\
\hline 3 Hospital & $5.0(8)$ \\
\hline \multicolumn{2}{|c|}{ Karnofsky Performance Status } \\
\hline$<70$ & $20.8(33)$ \\
\hline $70-80$ & $55.3(88)$ \\
\hline $90-100$ & $23.9(38)$ \\
\hline \multicolumn{2}{|c|}{ Tumor size $(\mathrm{cm})$} \\
\hline$<5.0$ & $54.1(86)$ \\
\hline$\geq 5.0$ & $45.9(73)$ \\
\hline \multicolumn{2}{|c|}{ Tumor pathology } \\
\hline GBM & $71.7(114)$ \\
\hline AA & $28.3(45)$ \\
\hline \multicolumn{2}{|l|}{ Tumor location } \\
\hline Frontal & $31.4(50)$ \\
\hline Parietal & $16.4(26)$ \\
\hline Temporal & $30.8(49)$ \\
\hline Occipital & $1.9(3)$ \\
\hline Other & $19.5(31)$ \\
\hline
\end{tabular}

$G B M$ glioblastoma, $A A$ anaplastic astrocytoma

inclusion in this analysis. Of the 159 patients, 114 had GBM and 45 had AA (Table 1). Nearly two-thirds of patients were 50 years of age or older and approximately $54 \%$ had tumors with a diameter less than $5.0 \mathrm{~cm}$. Ninetyfive percent of all patients had a neurologic functional class of 1 or 2 and $79 \%$ had KPS equal to 70 or greater. The most common of the 13 neurologic signs and symptoms experienced in our cohort were headache $(42.1 \%)$, impaired speech $(29.6 \%)$, abnormal mental status $(27.7 \%)$, and seizures $(26.4 \%)$. Treatment-related characteristics are listed in Table 2. Approximately $64 \%$ of patients underwent a
Table 2 Treatment characteristics

\begin{tabular}{ll}
\hline Characteristic & Percentage of assessable patients \\
\hline Extent of surgery & \\
Gross total resection & $33.9(54)$ \\
Subtotal resection & $30.2(48)$ \\
Biopsy only & $35.9(57)$ \\
Total RT dose (Gy) & \\
$\leq 54.4$ & $12.6(20)$ \\
$>54.4$ & $87.4(139)$ \\
\hline
\end{tabular}

partial (STR) or gross total resection (GTR) and 87\% received a total radiation dose in excess of 54.4 Gy (range 28-68 Gy). KPS was assigned by the treating physicians (radiation oncologist or neuro-oncologist) in 123 of 159 patients. In the remainder, KPS was assigned based on application of the standard scale to all available clinical records from the time of initial radiation oncology and/or neurologic oncology evaluation. The overall median survival was 14.9 months (range, 1.54-83.7 months).

Survival by RTOG-RPA risk group

Each of the 159 patients was assigned to one of the six risk classes as dictated by the pretreatment and treatmentrelated prognostic markers in the RTOG-RPA risk tree (Fig. 1). The median survival and 2-year survival rates are presented by RTOG-RPA group (Table 3). In terms of median survival, the risk groups retained their relative

Table 3 Survival by RTOG-RPA risk class in our cohort (A) compared to Curran et al. [3] (B)

\begin{tabular}{lll}
\hline $\begin{array}{l}\text { Class (number } \\
\text { of patients) }\end{array}$ & $\begin{array}{l}\text { Median survival, } \\
\text { months (IQ range) }\end{array}$ & $\begin{array}{l}\text { 2-Year } \\
\text { survival \% }\end{array}$ \\
\hline (A) & & \\
I (25) & $31.0(18.7-47.2)$ & 93.8 \\
II (7) & $27.5(16.4-45.6)$ & 66.7 \\
III (8) & $25.0(17.4-32.0)$ & 66.7 \\
IV (40) & $17.7(12.4-23.7)$ & 31.3 \\
V (62) & $10.8(5.9-16.9)$ & 11.5 \\
VI (17) & $6.4(4.9-11.2)$ & 6.3 \\
(B) & & \\
I (139) & 58.6 & 76 \\
II (34) & 37.4 & 68 \\
III (175) & 17.9 & 35 \\
IV (457) & 11.1 & 6 \\
V (395) & 8.9 & 4 \\
VI (263) & 4.6 & 6 \\
\hline
\end{tabular}

RTOG-RPA risk groups II and IV were excluded from further analysis due to small sample size 


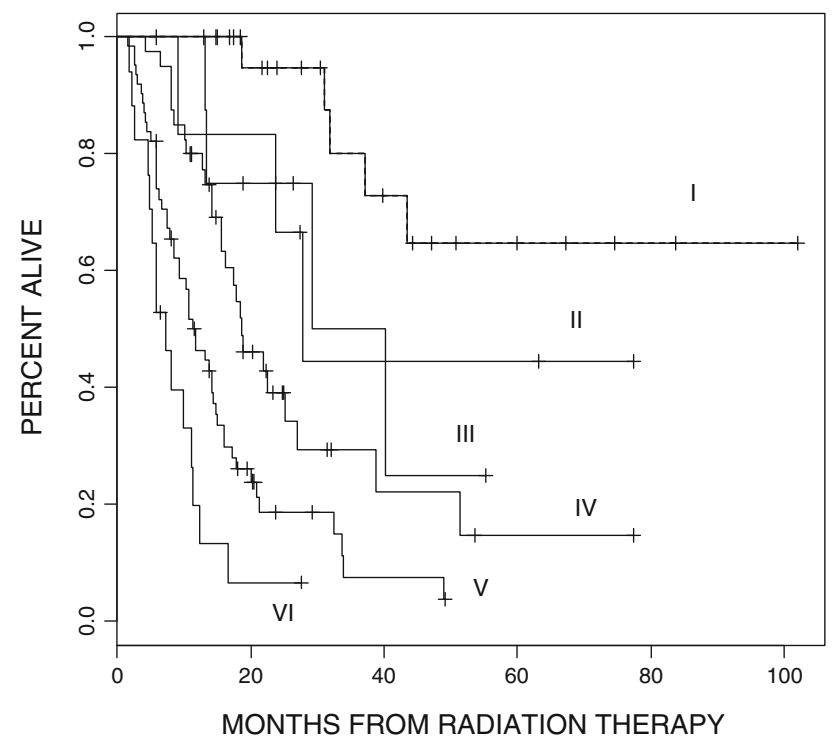

Fig. 2 Kaplan-Meier survival analysis by RTOG-RPA risk classes I-VI

prognostic ordering. In addition, the overall difference in survival curves was highly significantly different $\left(P<0.01, \chi^{2}\right.$ statistic $\left.=70\right)$ across all risk groups (Fig. 2$)$. In terms of 2-year survival and overall survival curves across all time points, the prognostic ordering was also retained with the exception of the survival curves of groups II $(n=7)$ and III $(n=8)$ which crossed at three time points. However, risk group II had a higher survival percentage at last follow-up.

\section{Recursive partitioning by RTOG-RPA predictors}

All 159 patients were newly recursively partitioned based on only the treatment and pre-treatment variables in the published RTOG-RPA. In the original RTOG-RPA, the most significant split was at age 50. In the present cohort, the most significant split was KPS (Fig. 3). Those who had KPS 90 or greater had a median survival of 25.1 months and those with KPS worse than 90 had a median survival of 11.8 months. For those with KPS 90 or better, the next split was histology. Patients with KPS $\geq 90$ and GBM had a median survival of 21.3 months whereas those with KPS $\geq 90$ and AA had a median survival of 27.7 months. For those who had KPS below 90, the next splits were at KPS 70 followed by age 50 .

The recursive partitioning process produced seven terminal nodes. By the log-rank test, non-significant survival differences were identified between three terminal node pairs. Following consolidation of these nodes, four risk classes remained with median survivals of 29.2, 21.1, 14.6, and 8.0 months, respectively. The corresponding 2-year actuarial survival rates were $100,48,20$, and $2.1 \%$. The Kaplan-Meier survival curves of the four remaining risk classes were highly significantly different from each other $\left(P<0.001, \chi^{2}\right.$ statistic $=111$, Fig. 4$)$.

\section{Recursive partitioning with expanded predictors}

All 159 patients were again recursively partitioned. However, this analysis was completed with the predictors in the RTOG-RPA plus several additional treatment and
Fig. 3 Recursive partitioning of 159 patients using the eight prognostic variables in RTOGRPA. Seven terminal nodes were combined into four significantly different survival classes $(P<0.001)$. The survival outcomes are displayed below the corresponding risk class. KPS Karnofsky Performance Status

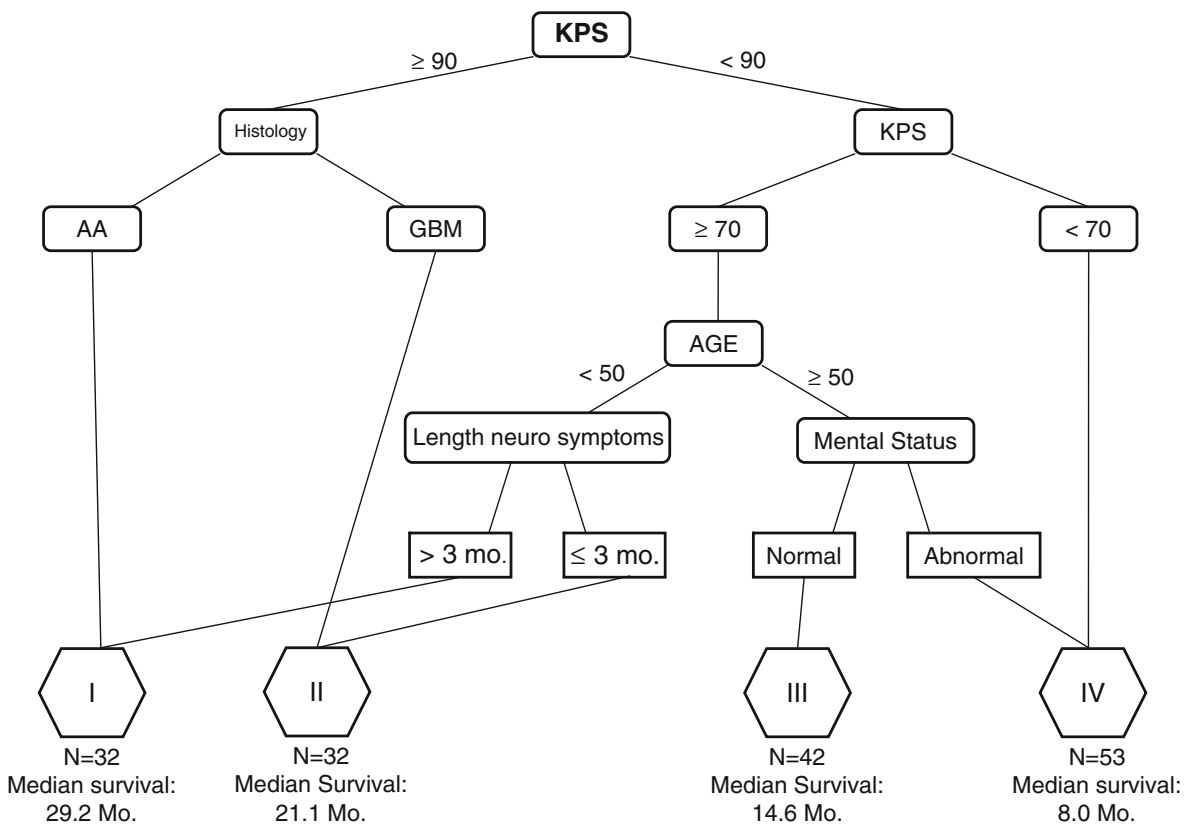




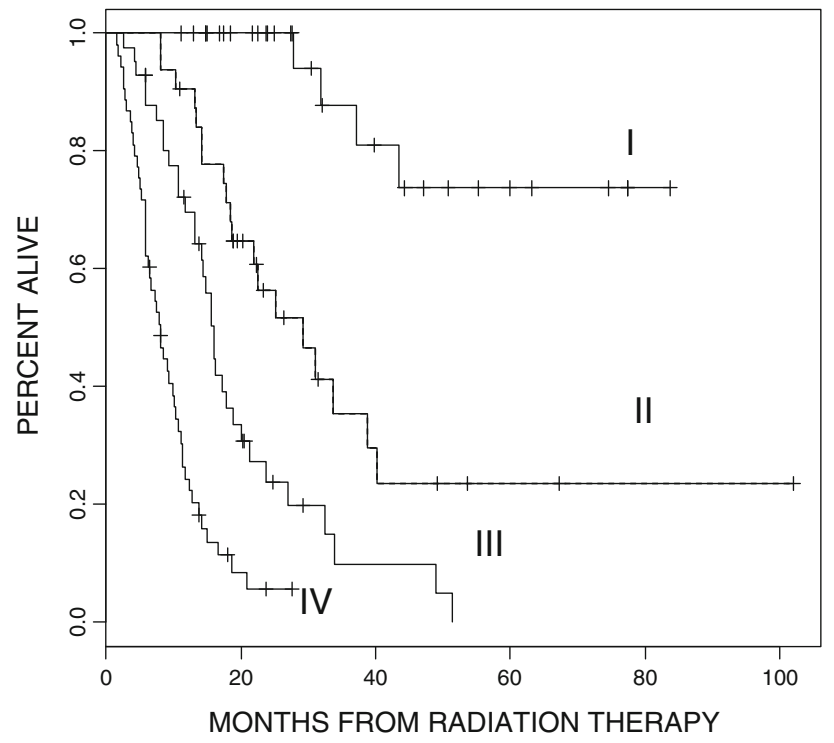

Fig. 4 Kaplan-Meier survival analysis of four risk classes resulting from new recursive partitioning of our cohort $(N=159)$ by the variables in RTOG-RPA

pre-treatment variables. In this analysis, the most significant factor was KPS of 90 (Fig. 5). For those with KPS 90 or greater, the next most significant factor was histology.
Patients with KPS $\geq 90$ and AA were further split based on the anatomic location of their tumor. Patients with AA, KPS $\geq 90$, and tumors located in the frontal lobe $(n=15$, median survival 39.8 months) had significantly better survival than those with $\mathrm{KPS} \geq 90$, AA, and tumors located elsewhere in the brain $(n=9$, median survival 27.5 months), contributing to risk classes I and II, respectively.

The recursive partitioning process produced 10 terminal nodes. By the log-rank test, non-significant survival differences were identified between three terminal node pairs. Following consolidation of these nodes, six risk classes remained with median survivals of $39.8,25.0,21.3,14.8$, 8.0, and 5.2 months, respectively. The corresponding 2 -year survival rates were $100,73,63,21,4.6$, and $0 \%$. The Kaplan-Meier survival curves of these six remaining classes were highly significantly different from each other $\left(P<0.001, \chi^{2}=147\right.$, Fig. 6).

\section{Discussion}

Multiple clinical trials of TMZ in combination with radiation therapy (RT) have provided clear evidence that its

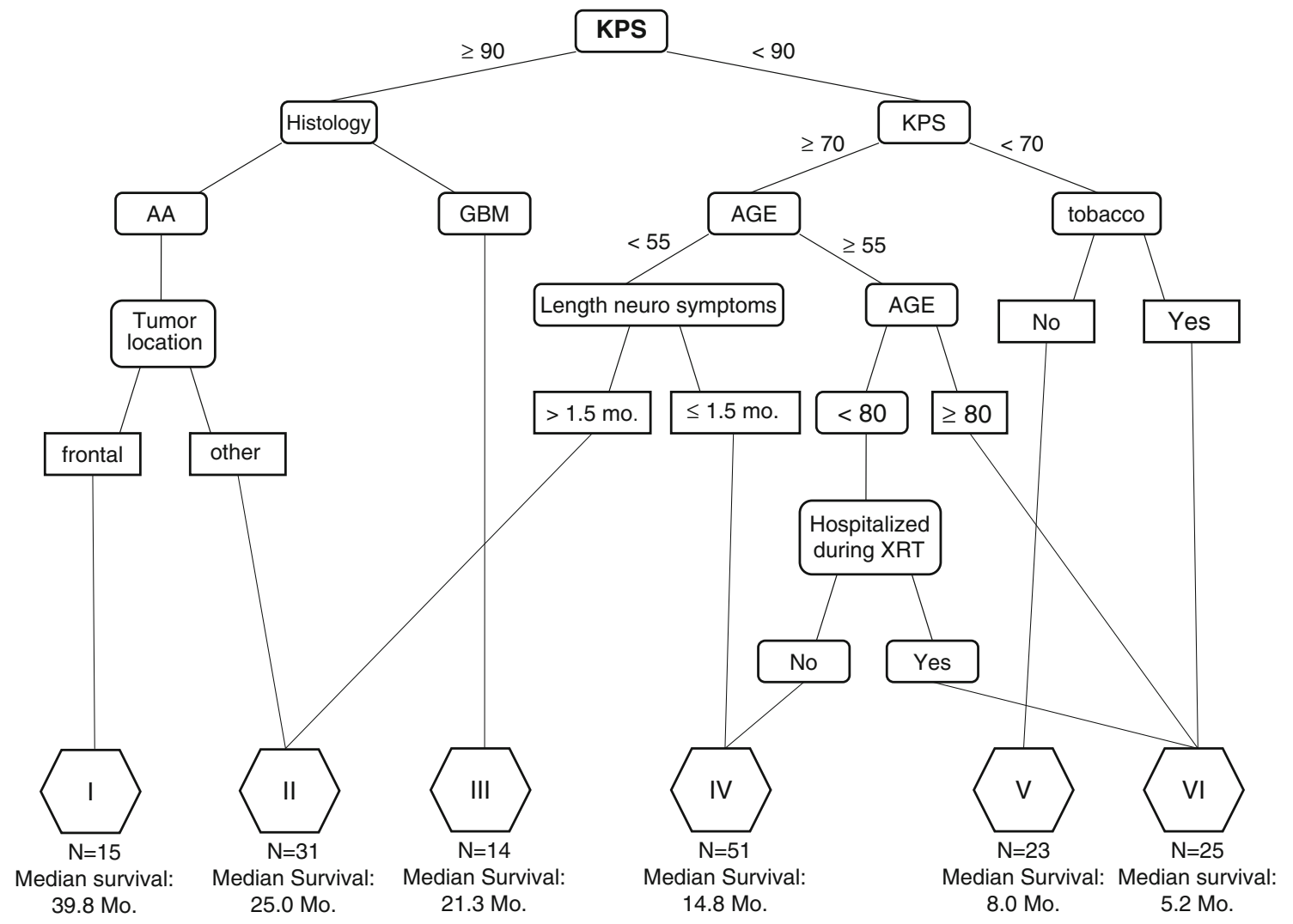

Fig. 5 Recursive partitioning of 159 patients using the expanded set of prognostic variables. Ten terminal nodes were combined into six significantly different survival classes $(P<0.01)$. The survival

outcomes are displayed below the corresponding risk class. KPS Karnofsky Performance Status, XRT radiation therapy 


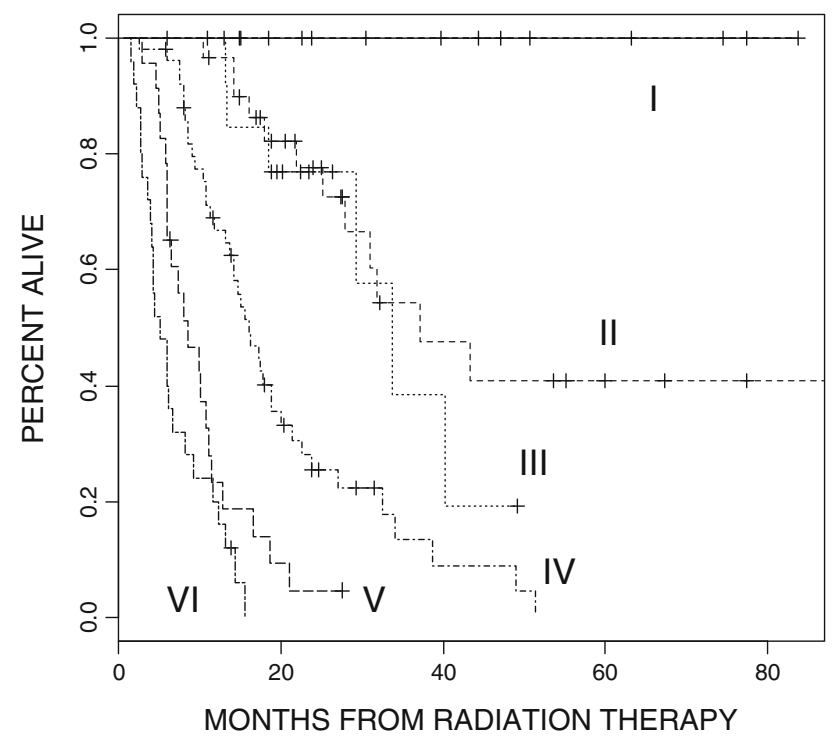

Fig. 6 Kaplan-Meier survival analysis of six risk classes resulting from recursive partitioning of our cohort $(N=159)$ by the variables in RTOG-RPA plus an expanded set of predictors

addition to RT after optimal surgical resection provides a clinically significant survival advantage compared to definitive or postoperative RT alone for high-grade glioma [11, 16-18]. However, neither these trials nor prior trials of other adjuvant treatments have shown as great an impact on survival as the pre-treatment and treatment-related factors in the RTOG-RPA risk classes [3]. Because concurrent and adjuvant TMZ have since become the standard of care for high-grade gliomas, we sought to validate the RTOG-RPA in a cohort of patients who were all treated with IMRT and TMZ. The continued validation of RTOG-RPA is highly valuable because it has been a critical tool in the design and interpretation of phase II trials and for stratification in phase III trials [4-14].

By assigning RTOG-RPA risk class to each patient in our cohort, we found these classes retained their relative prognostic significance. This result is similar to the partial validation of a modified version of the RTOG-RPA (classes III, IV, V) by the European Organization for Research and Treatment of Cancer (EORTC) via secondary analysis of the EORTC 26981/22981-NCIC CE3 phase III randomized trial of radiation therapy and concurrent and adjuvant TMZ $[17,22]$. In the EORTC study, median survival times (17, 15 and 10 months for classes III-V, respectively) were comparable to those of the corresponding RTOG-RPA risk classes in our cohort. However, the key distinction between the modified EORTC-RPA and the RTOG-RPA used in our analysis is the performance status and mental status scales used in the underlying clinical trials. In addition to examining only classes III-V, another difference between the EORTC validation and our analysis is that their partial validation was conducted from a cohort in which only approximately $50 \%$ of the patients received TMZ. Furthermore, all patients in the EORTC analysis had GBM and all were treated with 3D-conformal radiation therapy rather than IMRT.

Considering the total size of the present cohort $(N=159)$, validation of RTOG-RPA not surprisingly resulted in a limited number of patients in risk class II and III ( $n=7$ and $n=8$, respectively) and in some variation in the ordering of the survival curves of those classes. However, consistent with RTOG-RPA, the median survival of risk class II was longer than that of risk class III, as was its survival curve during the majority of follow-up time. For all other risk classes, the survival results retained the prognostic ordering of RTOG-RPA throughout the followup period. In our analysis, risk classes III-VI had greater survival than the corresponding classes in the original RTOG-RPA analysis by Curran et al. [3]. Similarly, in the EORTC-modified partial RPA validation, patients who received TMZ had longer median and 2-year survival than those who received RT only in classes III-V [22]. Somewhat surprisingly, classes I and II had shorter survival than in the original series, although the likelihood of such a finding is increased by the overall size of this cohort.

Another, more recently published partial validation of the RTOG-RPA also focused only on the classification of GBM patients (AA excluded). In this study, all patients were derived from the RTOG glioma database and all received radiation plus carmustine in trials from 1974 to 1995 [23]. This analysis revealed that modifications to RTOG-RPA classes III-VI did not increase the explained variation of survival in their cohort. However, the authors favored combining classes V and VI, leaving only four prognostic factors (age, performance status, extent of resection, and neurologic function), to simplify the application of RPA to GBM patients despite achieving no improvement in prognostic performance. Applying this simplified model to the RTOG glioma database, produced median survival times (17.1, 11.2 and 7.5 months for risk classes III, IV, and V + VI, respectively) that are also comparable to the corresponding RTOG-RPA risk classes in our cohort. The recent RTOG and EORTC validations $[22,23]$ and our analysis which together are comprised of data collected over decades in which radiation therapy, chemotherapy, and diagnostic techniques have advanced greatly indicate that the pretreatment patient and tumor characteristics initially described and organized by Curran et al. [3] likely continue to have a greater impact on outcome than do most treatment factors.

Several cooperative group trials and single-institution analyses of high-grade gliomas have identified performance status, histopathology (GBM or AA), and patient age as prognostic markers of patient survival with and without the use of recursive partitioning techniques $[3,7$, 
22-26]. Our results are consistent with these past findings. However, compared to the RTOG-RPA, new recursive partitioning of our cohort based on the treatment and pretreatment prognostic markers in RTOG-RPA, resulted in four significantly different risk classes with KPS rather than age as the most prognostic or initial branch point of the risk tree. Consistent with RTOG-RPA, onset of neurological symptoms greater than 3 months prior to surgery had a protective prognostic role and has been speculated to reflect relatively less aggressive disease [3]. Survival analysis based on these four risk groups produced greater separation of each groups' Kaplan-Meier curve compared to those produced by assigning RTOG-RPA class to each patient in our cohort. This increased prognostic power is quantified in the greater $\chi^{2}$ statistic (111 compared to 70 , respectively). In both the recently published RTOG analysis [23] and another recursive partitioning analysis limited to GBM patients enrolled in clinical trials prior to the TMZ era [7], the importance of KPS at presentation and patient age as prognostic markers was also seen. Furthermore, similar to our results, the impact of stratification of KPS at 70 on survival has also been described in the neurosurgical literature $[27,28]$.

The new recursive partitioning of our cohort based both on treatment and pre-treatment variables in RTOG-RPA in addition to the several additional variables outlined above, similarly resulted in KPS as the most significant or initial branch point of the risk tree. Some additional prognostic markers that do not appear in RTOG-RPA include tumor location, hospital admission during radiation therapy treatment, and history of tobacco use. While there is some controversy regarding the survival benefit of cytoreductive surgery prior to radiation therapy and TMZ [29-31], our finding that tumor location (frontal lobe) partitioned those with relatively good prognosis into risk groups I or II, is an interesting finding that could be further evaluated in a larger cohort. However, while tumor location has been reported to predict survival in prior analyses $[11,15]$, the number of patients subject to this decision point would be small in most clinical trials. The appearance of tobacco use as a marker of worse survival in malignant glioma patients with poorfunctional status is new and as such is subject to uncertainty regarding its prognostic value. Secondary recursive partitioning analysis of multi-institutional clinical trials of glioma patients from the TMZ era could potentially suggest the need for adjustments to RTOG-RPA or confirm the superior prognostic power of RTOG-RPA in its current form.

Although this is a retrospective review spanning 5 years and as such is subject to all the usual limitations, approximately one-third of these patients received TMZ on prospective clinical trials and thus underwent more rigorous monitoring in follow-up. Additional limitations of this study are inherent to the exploratory nature of recursive partitioning analysis. While this statistical technique has gained popularity in recent years because it can place patients in clearly definable risk categories, it is a nonparametric test with limitations. A statement of the probability that patients in a particular terminal node will survive to a given date cannot be made because of the nonparametric and post-hoc nature of this technique. In addition, the precise pattern of selection of prognostic factors can vary with only small changes in the group under study. This can occur if two or more variables have very similar prognostic power or are highly correlated. Lastly, the recursive partitioning technique may split a risk group on a variable that creates terminal nodes with survival distributions that are not significantly different from each other. However, such variables could be significant predictors of survival via traditional survival analyses.

Since the creation of the RTOG-RPA, it has been increasingly recognized that not all patients with highgrade glioma have the same prognosis. Interest in prognostically-important molecular markers and the development of targeted therapies is now emerging, as evident in several recent series [32-37]. In addition to the partial RPA validation, three nomograms were produced from the EORTC/NCIC clinical trial to predict outcomes for individual patients with newly diagnosed GBM [34]. An advantage of these nomograms is the potential for more individualized and more accurate prediction of a particular patient's survival than can be obtained from RPA. However, the major limitation of the nomograms is that their prognostic utility has yet to be validated in separate independent datasets. In the nomogram for patients who received RT and TMZ, the same prognostic factors as those in RTOG-RPA were recognized (performance status, age, mental status, and extent of resection). However, the EORTC/NCIC glioma clinical trial dataset allowed for the creation of a nomogram including additional prognostic factors not examined in the RTOG RPA model such as MGMT methylation status which was first established by Hegi et al. [35] to significantly impact outcomes of patients receiving alkylating chemotherapy like TMZ.

In addition to MGMT methylation status, another area of active investigation is gene profiling using DNA microarrays to identify gene expression patterns that may classify glioma tumors into prognostic groups [38]. From this analysis, AA has been separated into three prognostic subclasses corresponding to the stages in neurogenesis: proneural, proliferative, and mesenchymal. Recurrent tumors displayed a tendency to shift toward the mesenchymal subclass which was shown to have the worst prognosis [38].

More recently, biomarkers such as PTEN and EGFR have been investigated as potential prognostic markers in GBM. Also whole-genome sequencing of GBM samples has identified an $\mathrm{R} 132 \mathrm{H}$ (arginine to histidine) somatic 
mutation in the isocitric dehydrogenase-1 (IDHI) gene that has been shown to be present in more than $80 \%$ of grade II and grade III gliomas [39-43]. Subsequent studies have implicated the IDH1 R132H mutation in the early pathogenesis of glioma by acting in a dominant-negative manner to produce an approximately 100-fold elevation in levels of 2-hydroxyglutarate, a known onco-metabolite in human glioma samples [39-43]. Further elucidation of this pathway may establish serum markers for earlier diagnosis of low-grade glioma prior to malignant transformation or novel targets for chemotherapeutic intervention. Once the prognostic utility of each of the above markers is more fully understood, additional modifications to RPA or the EORTC nomograms will likely be required. The collection of these studies suggests that more precise estimates of patients' risks soon after diagnosis based on both clinical predictors and some combination of molecular markers will be possible and that eventually customized, targeted treatments have the potential to be established for highgrade glioma.

Our validation of RTOG-RPA suggests that it maintains prognostic significance and should continue to be used to predict individual patient outcomes for those treated with IMRT and TMZ and for interpretation and stratification of clinical trials. Although new recursive partitioning of our cohort indicates that KPS may be more prognostic in patients treated with TMZ than it was in RTOG-RPA, the relatively small size $(N=159)$ of our cohort compared to the original analysis by Curran et al. [3] suggests potential modifications to this classification that should be further explored through analysis of a larger cohort.

Acknowledgments The contribution to this manuscript by Anthony J. Paravati was supported by the Doris Duke Clinical Research Fellowship. Contributions by Douglas Landsittel and Yi-Fan Chen were supported by the University of Pittsburgh Clinical and Translational Science Institute Supplement 3 UL1 RR024153-04S4.

Funding Doris Duke Charitable Foundation. University of Pittsburgh Clinical and Translational Science Institute Supplement 3 UL1 RR024153-04S4.

\section{Conflict of interest None.}

Open Access This article is distributed under the terms of the Creative Commons Attribution Noncommercial License which permits any noncommercial use, distribution, and reproduction in any medium, provided the original author(s) and source are credited.

\section{References}

1. Laperriere N, Zuraw L, Cairncross G (2002) Radiotherapy for newly diagnosed malignant glioma in adults: a systematic review. Radiother Oncol 64(3):259-273

2. Stewart LBS (2002) Glioma Meta-analysis Trialists Group (GMT). Chemotherapy for high-grade glioma. Cochrane
Database Syst Rev. Issue 4. Art. No. CD003913. doi:10.1002/ 14651858.CD003913

3. Curran WJ Jr, Scott CB, Horton J, Nelson JS, Weinstein AS, Fischbach AJ, Chang CH, Rotman M, Asbell SO, Krisch RE et al (1993) Recursive partitioning analysis of prognostic factors in three Radiation Therapy Oncology Group malignant glioma trials. J Natl Cancer Inst 85(9):704-710

4. Scott CB, Scarantino C, Urtasun R, Movsas B, Jones CU, Simpson JR, Fischbach AJ, Curran WJ Jr (1998) Validation and predictive power of Radiation Therapy Oncology Group (RTOG) recursive partitioning analysis classes for malignant glioma patients: a report using RTOG 90-06. Int J Radiat Oncol Biol Phys 40(1):51-55

5. Coughlin C, Scott C, Langer C, Coia L, Curran W, Rubin P (2000) Phase II, two-arm RTOG trial (94-11) of bischloroethylnitrosourea plus accelerated hyperfractionated radiotherapy $(64.0$ or $70.4 \mathrm{~Gy})$ based on tumor volume ( $>20$ or $<$ or $=20 \mathrm{~cm}(2)$, respectively) in the treatment of newly-diagnosed radiosurgeryineligible glioblastoma multiforme patients. Int J Radiat Oncol Biol Phys 48(5):1351-1358

6. Del Rowe J, Scott C, Werner-Wasik M, Bahary JP, Curran WJ, Urtasun RC, Fisher B (2000) Single-arm, open-label phase II study of intravenously administered tirapazamine and radiation therapy for glioblastoma multiforme. J Clin Oncol 18(6):1254-1259

7. Lamborn KR, Chang SM, Prados MD (2004) Prognostic factors for survival of patients with glioblastoma: recursive partitioning analysis. Neuro Oncol 6(3):227-235

8. Langer CJ, Ruffer J, Rhodes H, Paulus R, Murray K, Movsas B, Curran W (2001) Phase II radiation therapy oncology group trial of weekly paclitaxel and conventional external beam radiation therapy for supratentorial glioblastoma multiforme. Int J Radiat Oncol Biol Phys 51(1):113-119

9. Miralbell R, Mornex F, Greiner R, Bolla M, Storme G, Hulshof M, Bernier J, Denekamp J, Rojas AM, Pierart M, van Glabbeke M, Mirimanoff RO (1999) Accelerated radiotherapy, carbogen, and nicotinamide in glioblastoma multiforme: report of European Organization for Research and Treatment of Cancer trial 22933. J Clin Oncol 17(10):3143-3149

10. Sarkaria JN, Mehta MP, Loeffler JS, Buatti JM, Chappell RJ, Levin AB, Alexander E III, Friedman WA, Kinsella TJ (1995) Radiosurgery in the initial management of malignant gliomas: survival comparison with the RTOG recursive partitioning analysis. Radiation Therapy Oncology Group. Int J Radiat Oncol Biol Phys 32(4):931-941

11. Stupp R, Dietrich PY, Ostermann Kraljevic S, Pica A, Maillard I, Maeder P, Meuli R, Janzer R, Pizzolato G, Miralbell R, Porchet F, Regli L, de Tribolet N, Mirimanoff RO, Leyvraz S (2002) Promising survival for patients with newly diagnosed glioblastoma multiforme treated with concomitant radiation plus temozolomide followed by adjuvant temozolomide. J Clin Oncol 20(5):1375-1382

12. Sultanem K, Patrocinio H, Lambert C, Corns R, Leblanc R, Parker W, Shenouda G, Souhami L (2004) The use of hypofractionated intensity-modulated irradiation in the treatment of glioblastoma multiforme: preliminary results of a prospective trial. Int J Radiat Oncol Biol Phys 58(1):247-252

13. Videtic GM, Gaspar LE, Zamorano L, Fontanesi J, Levin KJ, Kupsky WJ, Tekyi-Mensah S (1999) Use of the RTOG recursive partitioning analysis to validate the benefit of iodine- 125 implants in the primary treatment of malignant gliomas. Int J Radiat Oncol Biol Phys 45(3):687-692

14. Videtic GM, Gaspar LE, Zamorano L, Stitt LW, Fontanesi J, Levin KJ (2001) Implant volume as a prognostic variable in brachytherapy decision-making for malignant gliomas stratified by the RTOG recursive partitioning analysis. Int J Radiat Oncol Biol Phys 51(4):963-968 
15. Fisher B, Won M, Macdonald D, Johnson DW, Roa W (2002) Phase II study of topotecan plus cranial radiation for glioblastoma multiforme: results of Radiation Therapy Oncology Group 9513. Int J Radiat Oncol Biol Phys 53(4):980-986

16. Stupp R, Hegi ME, Mason WP, van den Bent MJ, Taphoorn MJ, Janzer RC, Ludwin SK, Allgeier A, Fisher B, Belanger K, Hau P, Brandes AA, Gijtenbeek J, Marosi C, Vecht CJ, Mokhtari K, Wesseling P, Villa S, Eisenhauer E, Gorlia T, Weller M, Lacombe D, Cairncross JG, Mirimanoff RO (2009) Effects of radiotherapy with concomitant and adjuvant temozolomide versus radiotherapy alone on survival in glioblastoma in a randomised phase III study: 5-year analysis of the EORTC-NCIC trial. Lancet Oncol 10(5):459-466

17. Stupp R, Mason WP, van den Bent MJ, Weller M, Fisher B, Taphoorn MJ, Belanger K, Brandes AA, Marosi C, Bogdahn U, Curschmann J, Janzer RC, Ludwin SK, Gorlia T, Allgeier A, Lacombe D, Cairncross JG, Eisenhauer E, Mirimanoff RO (2005) Radiotherapy plus concomitant and adjuvant temozolomide for glioblastoma. N Engl J Med 352(10):987-996

18. Athanassiou H, Synodinou M, Maragoudakis E, Paraskevaidis M, Verigos C, Misailidou D, Antonadou D, Saris G, Beroukas K, Karageorgis P (2005) Randomized phase II study of temozolomide and radiotherapy compared with radiotherapy alone in newly diagnosed glioblastoma multiforme. J Clin Oncol 23(10): 2372-2377

19. Team RDC (2010) R: a language and environment for statistical computing. R Foundation for Statistical Computing, Vienna

20. Therneau TM, Atkinson EJ (1997) An introduction to recursive partitioning using the RPART routines. Mayo Foundation, USA

21. LeBlanc M, Crowley J (1992) Relative risk trees for censored survival data. Biometrics 48:411-425

22. Mirimanoff RO, Gorlia T, Mason W, Van den Bent MJ, Kortmann RD, Fisher B, Reni M, Brandes AA, Curschmann J, Villa S, Cairncross G, Allgeier A, Lacombe D, Stupp R (2006) Radiotherapy and temozolomide for newly diagnosed glioblastoma: recursive partitioning analysis of the EORTC 26981/22981-NCIC CE3 phase III randomized trial. J Clin Oncol 24(16):2563-2569

23. Li J, Wang M, Won M, Shaw EG, Coughlin C, Curran WJ Jr, Mehta MP Validation and simplification of the radiation therapy oncology group recursive partitioning analysis classification for glioblastoma. Int J Radiat Oncol Biol Phys. doi:10.1016/j. ijrobp.2010.06.012

24. Curran WJ Jr, Scott CB, Nelson JS, Weinstein AS, Phillips TL, Murray K, Fischbach AJ, Yakar D, Schwade JG, Powlis WD et al (1992) A randomized trial of accelerated hyperfractionated radiation therapy and bis-chloroethyl nitrosourea for malignant glioma. A preliminary report of Radiation Therapy Oncology Group 83-02. Cancer 70(12):2909-2917

25. Deutsch M, Green SB, Strike TA, Burger PC, Robertson JT, Selker RG, Shapiro WR, Mealey J Jr, Ransohoff J II, Paoletti P et al (1989) Results of a randomized trial comparing BCNU plus radiotherapy, streptozotocin plus radiotherapy, BCNU plus hyperfractionated radiotherapy, and $\mathrm{BCNU}$ following misonidazole plus radiotherapy in the postoperative treatment of malignant glioma. Int J Radiat Oncol Biol Phys 16(6):1389-1396

26. Nelson DF, Curran WJ Jr, Scott C, Nelson JS, Weinstein AS, Ahmad K, Constine LS, Murray K, Powlis WD, Mohiuddin M et al (1993) Hyperfractionated radiation therapy and bis-chlorethyl nitrosourea in the treatment of malignant glioma-possible advantage observed at 72.0 Gy in 1.2 Gy B.I.D. fractions: report of the Radiation Therapy Oncology Group Protocol 8302. Int $\mathbf{J}$ Radiat Oncol Biol Phys 25(2):193-207

27. Devaux BC, O'Fallon JR, Kelly PJ (1993) Resection, biopsy, and survival in malignant glial neoplasms. A retrospective study of clinical parameters, therapy, and outcome. J Neurosurg 78(5): 767-775
28. Laws ER, Parney IF, Huang W, Anderson F, Morris AM, Asher A, Lillehei KO, Bernstein M, Brem H, Sloan A, Berger MS, Chang S (2003) Survival following surgery and prognostic factors for recently diagnosed malignant glioma: data from the Glioma Outcomes Project. J Neurosurg 99(3):467-473

29. Hess KR (1999) Extent of resection as a prognostic variable in the treatment of gliomas. J Neurooncol 42(3):227-231

30. Kreth FW, Warnke PC, Scheremet R, Ostertag CB (1993) Surgical resection and radiation therapy versus biopsy and radiation therapy in the treatment of glioblastoma multiforme. J Neurosurg 78(5):762-766

31. Quigley MR, Maroon JC (1991) The relationship between survival and the extent of the resection in patients with supratentorial malignant gliomas. Neurosurgery 29(3):385-388

32. Blough MD, Beauchamp DC, Westgate MR, Kelly JJ, Cairncross JG (2010) Effect of aberrant p53 function on temozolomide sensitivity of glioma cell lines and brain tumor initiating cells from glioblastoma. J Neurooncol. doi:10.1007/s11060-0100283-9

33. Costa BM, Caeiro C, Guimaraes I, Martinho O, Jaraquemada T, Augusto I, Castro L, Osorio L, Linhares P, Honavar M, Resende M, Braga F, Silva A, Pardal F, Amorim J, Nabico R, Almeida R, Alegria C, Pires M, Pinheiro C, Carvalho E, Lopes JM, Costa P, Damasceno M, Reis RM (2010) Prognostic value of MGMT promoter methylation in glioblastoma patients treated with temozolomide-based chemoradiation: a Portuguese multicentre study. Oncol Rep 23(6):1655-1662

34. Gorlia T, van den Bent MJ, Hegi ME, Mirimanoff RO, Weller M, Cairncross JG, Eisenhauer E, Belanger K, Brandes AA, Allgeier A, Lacombe D, Stupp R (2008) Nomograms for predicting survival of patients with newly diagnosed glioblastoma: prognostic factor analysis of EORTC and NCIC trial 26981-22981/CE.3. Lancet Oncol 9(1):29-38

35. Hegi ME, Diserens AC, Gorlia T, Hamou MF, de Tribolet N, Weller M, Kros JM, Hainfellner JA, Mason W, Mariani L, Bromberg JE, Hau P, Mirimanoff RO, Cairncross JG, Janzer RC, Stupp R (2005) MGMT gene silencing and benefit from temozolomide in glioblastoma. N Engl J Med 352(10):997-1003

36. Kitange GJ, Carlson BL, Schroeder MA, Decker PA, Morlan BW, Wu W, Ballman KV, Giannini C, Sarkaria JN (2010) Expression of CD74 in high grade gliomas: a potential role in temozolomide resistance. J Neurooncol. doi:10.1007/s11060-010-0186-9

37. van Nifterik KA, van den Berg J, van der Meide WF, Ameziane N, Wedekind LE, Steenbergen RD, Leenstra S, Lafleur MV, Slotman BJ, Stalpers LJ, Sminia P (2010) Absence of the MGMT protein as well as methylation of the MGMT promoter predict the sensitivity for temozolomide. Br J Cancer. doi:10.1038/sj.bjc.6 605712

38. Phillips HS, Kharbanda S, Chen R, Forrest WF, Soriano RH, Wu TD, Misra A, Nigro JM, Colman H, Soroceanu L, Williams PM, Modrusan Z, Feuerstein BG, Aldape K (2006) Molecular subclasses of high-grade glioma predict prognosis, delineate a pattern of disease progression, and resemble stages in neurogenesis. Cancer Cell 9(3):157-173

39. Dang L, White DW, Gross S, Bennett BD, Bittinger MA, Driggers EM, Fantin VR, Jang HG, Jin S, Keenan MC, Marks KM, Prins RM, Ward PS, Yen KE, Liau LM, Rabinowitz JD, Cantley LC, Thompson CB, Vander Heiden MG, Su SM (2009) Cancerassociated IDH1 mutations produce 2-hydroxyglutarate. Nature 462(7274):739-744

40. Ward PS, Patel J, Wise DR, Abdel-Wahab O, Bennett BD, Coller HA, Cross JR, Fantin VR, Hedvat CV, Perl AE, Rabinowitz JD, Carroll M, Su SM, Sharp KA, Levine RL, Thompson CB (2010) The common feature of leukemia-associated IDH1 and IDH2 mutations is a neomorphic enzyme activity converting alphaketoglutarate to 2-hydroxyglutarate. Cancer Cell 17(3):225-234 
41. Yan H, Bigner DD, Velculescu V, Parsons DW (2009) Mutant metabolic enzymes are at the origin of gliomas. Cancer Res 69(24):9157-9159

42. Parsons DW, Jones S, Zhang X, Lin JC, Leary RJ, Angenendt $\mathrm{P}$, Mankoo P, Carter H, Siu IM, Gallia GL, Olivi A, McLendon R, Rasheed BA, Keir S, Nikolskaya T, Nikolsky Y, Busam DA, Tekleab H, Diaz LA Jr, Hartigan J, Smith DR, Strausberg RL, Marie SK, Shinjo SM, Yan H, Riggins GJ, Bigner DD, Karchin
R, Papadopoulos N, Parmigiani G, Vogelstein B, Velculescu VE, Kinzler KW (2008) An integrated genomic analysis of human glioblastoma multiforme. Science 321(5897):1807-1812

43. Yan H, Parsons DW, Jin G, McLendon R, Rasheed BA, Yuan W, Kos I, Batinic-Haberle I, Jones S, Riggins GJ, Friedman H, Friedman A, Reardon D, Herndon J, Kinzler KW, Velculescu VE, Vogelstein B, Bigner DD (2009) IDH1 and IDH2 mutations in gliomas. N Engl J Med 360(8):765-773 